\title{
Les mesures anthroporadiamétriques dans les basses énergies : évolution technologique et bases des recherches actuelles
}

\author{
D. FRANCK, L. DE CARLAN, Ph. BÉRARD, C. DOUSSE, P. PIHET, \\ N. RAZAFINDRALAMBO, R. SOULIÉ
}

(Manuscrit requ le 17 mars 1997, révisé le 21 juin 1997, accepté le 15 octobre 1997)

RÉSUMÉ L'examen d'anthroporadiamétrie pulmonaire est par sa rapidité de réponse un moyen utile à la surveillance médicale pour l'estimation de la contamination après inhalation de produits radiotoxiques. Les actinides (en particulier ${ }^{239} \mathrm{Pu}$ ) sont des émetteurs $X$ et $\gamma$ de basse énergie. Malgré les améliorations réalisées en matière de détection par l'arrivée des détecteurs germanium de grand volume, les performances des installations montrent des sensibilités de détection encore beaucoup trop élevées comparées aux limites annuelles d'incorporation. L'amélioration de la mesure in vivo exige le développement d'un système de spectrométrie $X$ de résolution en énergie comparable à celle du germanium. Les critères de ces nouveaux détecteurs reposent sur le fonctionnement à température ambiante ainsi qu'une surface de détection adaptée au thorax. Parmi les matériaux possibles, les semiconducteurs $\mathrm{Si}$, CdTe ont été identifiés comme les plus prometteurs.

ABSTRACT $\mathbf{X}$ and low energy gamma ray spectroscopy for in-vivo monitoring: Technical evolution and requirements for future research.

Whole body counting remains a major tool for individual monitoring for its fast response to assess the contamination of the lung after inhalation of radiotoxic compounds. Considering actinides such as ${ }^{239} \mathrm{Pu}$ emitting associated $X$ and low energy $\gamma$ rays, in spite of improved detection methods based on large volume germanium crystals, all investigations show detection limits much too high compared to annual limit of incorporation. Based on results obtained using germanium systems, it was shown that the improvement of lung monitoring requires the development of $X$ spectroscopy systems with resolution comparable to germanium but operating at room temperature to allow a larger detection area better adjusted to the chest volume. Among possible materials, Si and CdTe semiconductors were identified as most promising ones.

\section{Introduction}

L'exposition interne suite à l'inhalation d'actinides est déterminée par examen anthroporadiamétrique pulmonaire et par les dosages sur excréta. Le suivi dosimétrique des travailleurs exposés en routine permet de garantir le respect 
des limites de dose imposées par la réglementation. Les installations opérationnelles doivent permettre la détection de très faibles niveaux de contamination, étant donné la radiotoxicité élevée des composés.

Parmi les méthodes possibles de mesure d'une contamination par des actinides émetteurs alpha, deux types d'examens sont prescrits : les analyses d'excrétion et la mesure de la rétention pulmonaire. L'anthroporadiamétrie ou spectrométrie in vivo, est l'outil privilégié de la surveillance médicale pour estimer la contamination pulmonaire après inhalation de produits radiotoxiques (Toohey et al., 1991). En effet, son intérêt est primordial compte tenu que, pour les composés peu transférables, le taux d'excrétion est faible et la dose engagée reste principalement due à celle de l'appareil respiratoire. De plus, l'évaluation dosimétrique est plus directe dans la mesure où les modèles d'excrétion et biocinétiques systémiques n'interviennent pas dans l'estimation de l'incorporation.

Aussi privilégiée soit-elle, l'anthroporadiamétrie n'en reste pas moins limitée, pour les actinides, par sa relativement faible sensibilité. Cette limitation est inhérente au principe de la mesure d'émetteurs alpha par l'intermédiaire des émissions $X$ et gamma associées. L'efficacité de détection est en effet diminuée par la faible intensité de ces émissions et par leur assez faible énergie entraînant une forte absorption dans les tissus traversés avant d'atteindre le détecteur. Ces difficultés sont importantes pour de nombreux isotopes dont les raies gamma sont émises dans l'intervalle de 50 à $200 \mathrm{keV}$. Elles deviennent critiques pour des isotopes comme ${ }^{239} \mathrm{Pu}$ dont les raies $\mathrm{X}$ et gamma sont émises entre 10 et $50 \mathrm{keV}$ avec des probabilités d'émissions très faibles (Tab. I). Dans ce dernier cas, l'anthroporadiamétrie pulmonaire est restée un défi. La mesure est en effet compliquée par la multiplicité des émissions $X$ dans le même intervalle d'énergie comme le montre le tableau I pour ${ }^{239} \mathrm{Pu}$ et ${ }^{240} \mathrm{Am}$.

Les mesures anthroporadiamétriques sont également utilisées en cas d'accident de contamination pour guider l'intervention sanitaire. Ici encore, la sensibilité des techniques utilisées, l'identification des contaminants et la précision de la mesure, sont capitales compte tenu des contraintes de temps pour fournir une première réponse fiable. La recherche doit être maintenue pour la sélection de nouveaux détecteurs et pour le développement de méthodes de surveillance individuelle performantes.

Ce rapport dresse le bilan de l'évolution suivie par les systèmes de spectrométrie pulmonaire basse énergie afin de faire ressortir les critères à prendre en compte pour tenter d'obtenir un gain de sensibilité significatif par le biais de nouvelles techniques de détection. Il s'appuie sur les résultats obtenus à l'aide de détecteurs au germanium pour montrer la corrélation, dans le cas d'une mesure $X$, entre limite de détection, incertitudes statistiques et erreurs systématiques d'étalonnage. Il illustre les orientations de recherche les plus prometteuses et les conditions qu'il est nécessaire de réunir pour faire aboutir cette recherche. 


\section{L'évolution des systèmes de mesure depuis 30 ans}

Le développement d'un système efficace de détection pour la mesure in vivo du plutonium retenu dans les poumons a fait depuis plus de 20 ans l'objet de nombreuses recherches, entre autres, (Morucci, 1966 ; Daburon et Rigaudière, 1976 ; Rundo et al., 1978 ; Ramsden et Foster, 1986 ; Bataller et Girard de Vasson, 1994).

\section{TABLEAU I}

Énergies et probabilités d'émission $\mathbf{P}(E)$ des principales raies $X$ et $\gamma$ de ${ }^{239} \mathrm{Pu}$ et de ${ }^{241} \mathrm{Am}$ utilisées pour la mesure anthroporadiamétrique (Bland $e t$ al., 1992).

Energies and intensities $\mathrm{P}(\mathrm{E})$ of $\mathrm{X}$ and $\gamma$-rays from ${ }^{239} \mathrm{Pu}$ and ${ }^{241} \mathrm{Am}$ decay used for in vivo measurement (Bland et al., 1992).

\begin{tabular}{|c|c|c|c|c|}
\hline Radiontelde & Nhlonium 289 & & Américiunn 241 & $n^{2}+y_{1}$ \\
\hline Émissions & Énergie & $P(E)(\%)$ & Énergie & $\mathrm{P}(\mathrm{E})(\%)$ \\
\hline raies $X$ & $\begin{array}{l}13,6 \\
17,0 \\
20,3\end{array}$ & $\begin{array}{l}1,65 \\
2,36 \\
0,56\end{array}$ & $\begin{array}{c}13,9 \\
17,5 \\
21\end{array}$ & $\begin{array}{r}13 \\
19 \\
4,8\end{array}$ \\
\hline raies $\gamma$ & 51,6 & 0,03 & $\begin{array}{l}26,3 \\
59,5\end{array}$ & $\begin{array}{c}2,4 \\
36\end{array}$ \\
\hline
\end{tabular}

\subsection{Les compteurs proportionnels}

Tandis que les scintillateurs étaient suffisants pour mesurer les radionucléides émettant des raies $\gamma$ d'énergie élevée comme les produits de fission, la mesure de certains actinides comme ${ }^{239} \mathrm{Pu}$ à de faibles niveaux d'activité a conduit au développement des compteurs proportionnels à gaz (CP) possédant une meilleure résolution et un bruit de fond beaucoup plus faible (Ehret et al.,

- 1964 ; Morucci, 1966). La résolution en énergie du CP était suffisante pour séparer les raies $\mathrm{X}$ de ${ }^{239} \mathrm{Pu}$ (Fig. 1). Cependant, malgré une surface de détection relativement élevée, ce détecteur était pénalisé par le manque de pouvoir d'absorption du gaz induisant une faible efficacité photoélectrique et limitant sa gamme dynamique à $50 \mathrm{keV}$. En outre, la dégradation de sa résolution avec le temps, probablement due à un vieillissement du mélange gazeux, et l'ajustement délicat de l'analyse d'impulsion en avait fait un système, certes intéressant sur le plan physique, mais instable du point de vue de l'application.

\subsection{Les détecteurs "phoswich"}

L'efficacité intrinsèque des compteurs proportionnels étant trop faible, des détecteurs de type phoswich ont été développés (Daburon et Rigaudière, 1976; Newton et al., 1978). Ce détecteur est un assemblage de deux scintillateurs, généralement un $\mathrm{NaI}$ de faible épaisseur $(1,5 \mathrm{~mm})$ associé à un cristal plus épais de CsI ( $50 \mathrm{~mm})$, permettant à la fois la détection basse énergie et la 
réduction du bruit de fond. Le phoswich présente l'avantage de posséder une efficacité de détection élevée en combinant l'usage du scintillateur et une grande surface de détection. Ceux-ci avaient en revanche l'inconvénient de posséder un faible pouvoir de résolution qui ne permettait pas de séparer les raies $\mathrm{X}$ de ${ }^{239} \mathrm{Pu}$ (Fig. 1).

\subsection{Les détecteurs germanium hyper pur $\mathrm{Ge}(\mathrm{HP})$}

Il y a un peu plus d'une dizaine d'années, grâce à la maîtrise des procédés de fabrication de monocristaux de plus en plus gros et de meilleure qualité, la spectrométrie utilisant des détecteurs germanium de haute pureté (Ge(HP)) s'est largement répandue pour la mesure d'échantillons. Ces détecteurs offrant une excellente résolution représentaient une alternative intéressante pour la mesure in vivo des actinides, y compris pour le plutonium (Falk et al., 1979 ; Palmer et Rieksts, 1984). La figure 1 représente les spectres obtenus dans des conditions de mesure similaires à l'aide des trois systèmes et résume les avantages et inconvénients des systèmes de détection existants pour la mesure pulmonaire de ${ }^{239} \mathrm{Pu}$ (Razafindralambo, 1995). Parmi ces techniques, on voit que le $\mathrm{Ge}(\mathrm{HP})$ représente la seule véritable avancée durant cette période puisqu'il allie une excellente résolution en énergie à une efficacité intrinsèque élevée sur une gamme dynamique allant de 10 à $200 \mathrm{keV}$. Cette propriété le rend particulièrement bien adapté à la détection des isotopes de l'uranium (Bérard et Aussel, 1994).

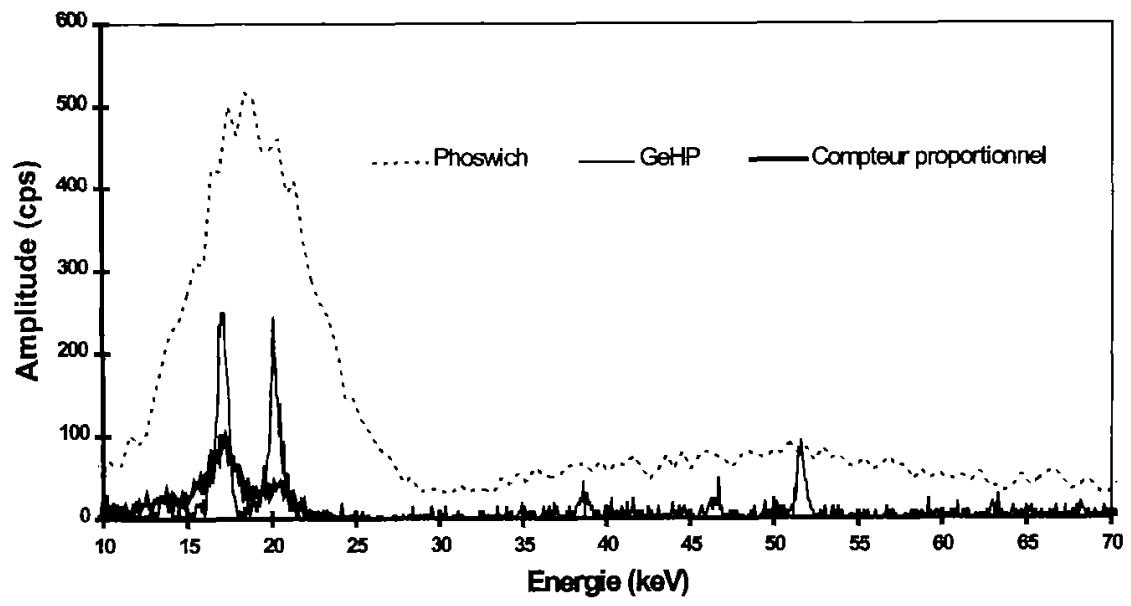

Fig. 1 - Comparaison des spectres de ${ }^{239} \mathrm{Pu}$ mesurés avec un fantôme de Livermore pour differents systèmes de détection : (i) Phoswich $\left(325 \mathrm{~cm}^{2}\right)$; (ii) compteur proportionnel $\left(250 \mathrm{~cm}^{2}\right)$; (iii) $\mathrm{Ge}(\mathrm{HP})\left(160 \mathrm{~cm}^{2}\right)$ (Razafindralambo, 1995).

Comparison of ${ }^{239} \mathrm{Pu}$ spectra measured with Livermore phantom using different in vivo systems: (i) Phoswich (325 $\left.\mathrm{cm}^{2}\right)$; (ii) proportional counter (250 $\left.\mathrm{cm}^{2}\right)$; (iii) $\mathrm{HPGe}$ $\left(160 \mathrm{~cm}^{2}\right)$ (Razafindralambo, 1995). 
Quelques constructeurs se sont lancés dans le développement de systèmes pulmonaires $\mathrm{U} / \mathrm{Pu}$ utilisant un groupement de détecteurs $\mathrm{Ge}(\mathrm{HP})$. Ces systèmes remplacent progressivement les compteurs proportionnels et les détecteurs de type phoswich dans les laboratoires de surveillance médicale. Développer de tels systèmes représente cependant une prouesse technologique car, si les détecteurs $\mathrm{Ge}(\mathrm{HP})$ sont aujourd'hui très fiables, ils réclament l'utilisation de cryostats encombrants et complexes. Cela a pour conséquence, qu'assez paradoxalement leurs excellentes propriétés intrinsèques n'ont au total pas permis de diminuer la limite de détection pour ${ }^{239} \mathrm{Pu}$, en particulier par rapport au phoswich. Néanmoins, les systèmes Ge(HP) demeurent à ce jour la référence pour la mesure pulmonaire du plutonium. Ils ont ouvert la voie vers d'autres solutions technologiques et recherches avec pour objectif d'une part la spectrométrie $\mathrm{X}$ de haute résolution, permettant notamment l'identification des pics par déconvolution (Bland et al., 1992) et l'analyse globale des spectres X et gamma (Razafindralambo et al., 1994), et, d'autre part, une réponse matricielle de la répartition de la contamination (4 à 8 détecteurs suivant les cas) (Bataller et al., 1994; Bérard et Aussel, 1994).

\section{Les systèmes $\mathrm{Ge}(\mathrm{HP})$ : un point de normalisation}

La limite de détection (LD) des détecteurs de spectrométrie est principalement déterminée par l'efficacité absolue $\left(\varepsilon_{\mathrm{a}}\right)$ et par le bruit de fond radiatif (Currie, 1968). Pour la mesure pulmonaire, $\varepsilon_{\mathrm{a}}$ est elle même déterminée par l'absorption des photons dans les poumons et les tissus extra thoraciques, et par la surface de détection (Pihet et al.,1995b). Aux faibles énergies X et $\gamma$, l'influence de ces paramètres sur la réponse du détecteur est déterminante. L'application de la LD dans ce cas, n'est pas triviale étant donné les erreurs systématiques inhérentes à cette mesure (Bérard et Aussel, 1994). Néanmoins, la LD déterminée dans des conditions bien définies est un paramètre utile pour comparer différents systèmes de mesures et pour estimer leurs niveaux de performance.

Le tableau II rappelle la valeur de LAI (limite annuelle d'incorporation) actuellement recommandée pour ${ }^{239} \mathrm{Pu}$ (composé de type $\mathrm{S}$ ) par la Commission Internationale de Protection Radiologique (CIPR). Comme on le voit, la LD d'un système $\mathrm{Ge}(\mathrm{HP})$ pour la mesure directe de ${ }^{239} \mathrm{Pu}^{(1)}$ dans des conditions de mesure de routine reste nettement plus élevée que la LAI. Les performances d'un système de détection doivent donc être évaluées en comparant la LD au produit de la quantité inhalée par la valeur de la rétention au moment de la mesure. Pour ${ }^{239} \mathrm{Pu}$, on est ainsi amené à comparer des quantités aussi faibles

(1) On ne considère pas ici le cas d'une mesure indirecte par le biais de la raie $\gamma$ de ${ }^{24 l} \mathrm{Am}$, méthode aussi utilisée lorsque la proportion de ${ }^{239} \mathrm{PU}$, et de ${ }^{241} \mathrm{Am}$ est supposée connue 
que $150 \mathrm{~Bq}$ à des $\mathrm{LD}$ qui restent typiquement de l'ordre de 3000 à $4000 \mathrm{~Bq}$ (Pihet et al., 1995a).

\section{TABLEAU II}

Comparaison des valeurs de LAI et de rétention pulmonaire correspondante avec la LD obtenue à l'aide d'un système de détecteurs Ge(HP) (CEA-Cadarache) utilisé pour la mesure de ${ }^{239} \mathrm{Pu}$.

Comparison of the ALI and the corresponding retention level with the detection limit obtained with a $\mathrm{Ge}(\mathrm{HP})$ system used for ${ }^{239} \mathrm{Pu}$ measurement.

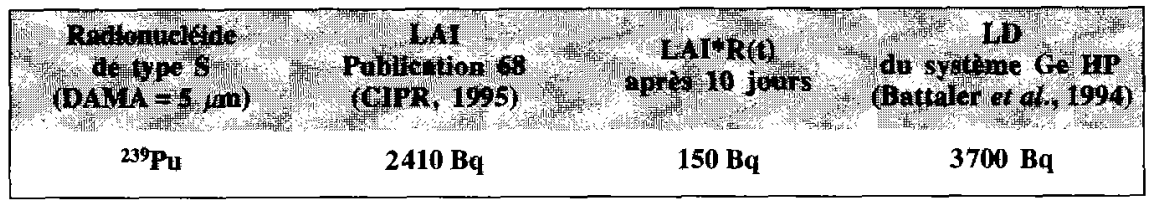

Même si les détecteurs Ge(HP) ont considérablement amélioré le pouvoir de résolution des systèmes de mesure et, par la même, l'identification des produits de contamination, des efforts importants sont nécessaires pour abaisser, le plus possible, la limite de détection. Même dans le cas le plus défavorable de ${ }^{239} \mathrm{Pu}$, une diminution significative (un facteur 2 à 3) de la LD devrait contribuer à pouvoir détecter plus tôt une contamination accidentelle. En outre, la résolution et le seuil de détection $(\mathrm{LD} / 2)$ doivent permettre d'affirmer l'absence de contamination (au risque $\alpha=0,025$ de se tromper) avec une beaucoup plus grande assurance.

Les premières études réalisées ont donc permis de comprendre ce qui contraint les $\mathrm{LD}$ des systèmes $\mathrm{Ge}(\mathrm{HP})$ à rester à des valeurs aussi élevées. Pour cela, la réponse de plusieurs de ces systèmes a été évaluée de manière systématique en fonction de différents paramètres, dans différentes conditions de source (ponctuelles, étalées et diffuses), d'activité (102 à 104 Bq) et d'absorption par les tissus.

Les résultats obtenus (Razafindralambo, 1995) montrent que la LD élevée des systèmes $\mathrm{Ge}(\mathrm{HP})$ pour la mesure de ${ }^{239} \mathrm{Pu}$ par ses raies UXL est liée principalement, malgré leur grande efficacité intrinsèque, à la diminution de l'efficacité de détection dont sont responsables :

- l'autoabsorption des rayons $\mathrm{X}$ dans le poumon qui entraîne à elle seule une diminution importante des raies de plus faible énergie. Ainsi la raie de 13,6 keV n'est plus détectée au-delà d'environ $3 \mathrm{~cm}$ d'épaisseur pulmonaire. Pour les raies de 17,2 et de $20,2 \mathrm{keV}$, la mesure est possible jusqu'à $10 \mathrm{~cm}$ d'épaisseur pulmonaire.

- l'épaisseur des tissus autour de la cage thoracique. Les côtes jouent également un effet d'écran réduisant la surface de détection d'environ 40 à $50 \%$. 
- la faible surface utile de détection contrainte par la place occupée par les détecteurs équipés de leurs cryostats. Ceux-ci réduisent fortement l'adaptabilité de l'installation.

Ces résultats amènent plusieurs remarques. Malgré la contrainte des cryostats, la LD d'un système Ge(HP) peut être substantiellement diminuée en optimisant la géométrie de mesure particulièrement critique en raison de l'absorption des raies de basse énergie. Dans les conditions définies d'un fantôme de Livermore, le gain d'efficacité peut atteindre $30 \%$. Par contre, le bruit de fond des détecteurs $\mathrm{Ge}(\mathrm{HP})$ est fixé par les conditions de mesure et ne peut pas être réduit, l'usage classique d'un détecteur en anticoïncidence étant interdit par les cryostats. Enfin, la conséquence évidente de ces caractéristiques est que la LD dépend de façon cruciale de la distribution de l'activité dans les poumons.

Cette étude a également révélé l'importance des incertitudes de mesure qui, à basse énergie, ne peuvent plus être négligées aussi simplement qu'en anthroporadiamétrie conventionnelle. Même si une technique d'étalonnage appropriée réduit l'importance des erreurs systématiques entre la géométrie du fantôme de référence et l'individu, il subsiste en effet de larges incertitudes (Tab. III) qui ont pu être estimées globalement autour de $80 \%$ ( 2 écarts-types), dues principalement à l'hétérogénéité de la rétention pulmonaire et à la difficulté de prendre en compte avec précision les paramètres morphologiques individuels. On notera néanmoins, que l'influence de l'épaisseur de tissu extrathoracique domine et peut être nettement diminuée par une mesure plus appropriée que l'utilisation de formules bioparamétriques. Les techniques utilisées (ultrasons, IRM) sont cependant relativement lourdes et ne sont utilisées à l'heure actuelle, que dans quelques laboratoires très spécialisés (Kang et al., 1993). Enfin, les incertitudes liées à l'hétérogénéité de la rétention pulmonaire sont d'autant plus grandes que les systèmes Ge(HP) sont constitués d'un nombre limité de détecteurs de relativement faible surface, focalisés puisqu'ils ne mesurent que les rayons $\mathrm{X}$ émis dans l'axe du cristal.

Pour terminer, si les détecteurs Ge(HP) apportent par leur excellente résolution $(600 \mathrm{eV})$ un pouvoir de résolution élevé pour séparer des raies interférentes, ils ne peuvent cependant pas séparer les raies $X$ de ${ }^{239} \mathrm{Pu}$ et de ${ }^{241} \mathrm{Am}$ étant donné les faibles différences d'énergie (Tab. I, Fig. 2). Ceci conduit en cas de contamination mixte à rechercher d'autres solutions basées sur la corrélation entre les raies $\mathrm{X}$ et les raies gamma de plus haute énergie. Ici encore, la courbe d'efficacité du détecteur $\mathrm{Ge}(\mathrm{HP})$ est un atout déterminant, difficile à retrouver dans d'autres systèmes.

Les systèmes Ge(HP) offrent donc une technologie performante et apportent des points de repères très utiles. Les limites constatées conduisent néanmoins à considérer que l'amélioration de la mesure pulmonaire de ${ }^{239} \mathrm{Pu}$ passe par le développement d'une nouvelle technique de détection à condition que celle-ci puisse permettre d'abaisser significativement la limite de détection et 
de réduire l'incertitude de mesure en trouvant le meilleur compromis entre les paramètres discutés plus haut.

TABLEAU III

Estimation des incertitudes sur les différents facteurs correctifs impliqués dans l'étalonnage des systèmes de mesure pulmonaire utilisant la spectrométrie basse énergie.

Valeurs observées pour la mesure de ${ }^{239} \mathrm{Pu}$ (Razafindralambo, 1995 ; Pihet et al., 1995a).

Assessement of the uncertainties on different correction factors involved in the calibration of lung monitoring systems using low energy spectroscopy. Values observed for the measurement of ${ }^{239} \mathrm{Pu}$ (Razafindralambo, 1995 ; Pihet et al., 1995a).

\section{5.}

Positionnement des détecteurs

Variation du volume pulmonaire

\section{Ecart typertitif}

9

4

19

11

3

13

33

* $S_{x} / x=(\Delta x / x) / 3$ avec $\Delta x / x$ égal à l'erreur maximale estimée.

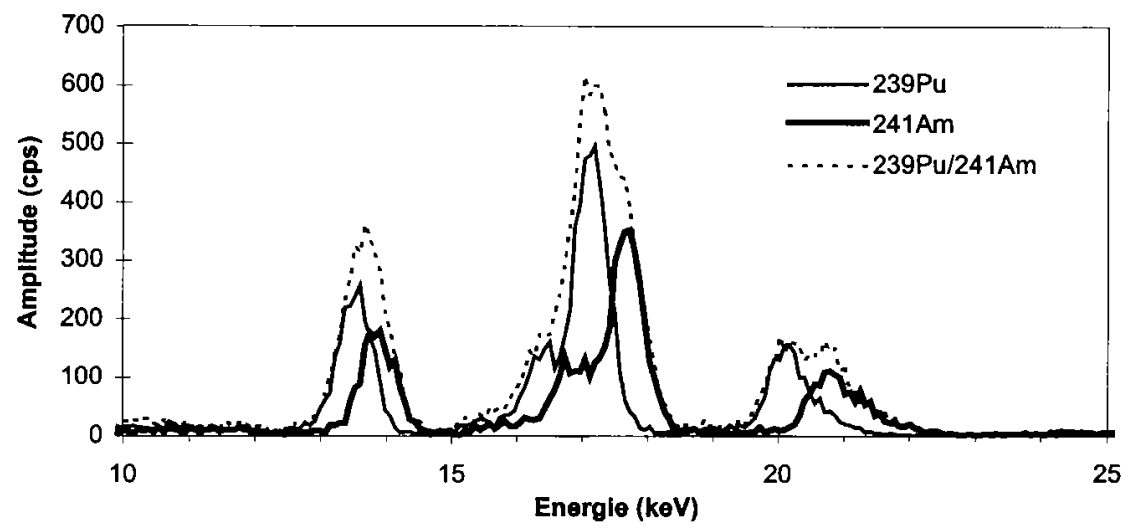

Fig. 2 - Interference des raies $U X L$ de ${ }^{239} P u$ et des raies $N p X L$ de ${ }^{241} A m$ (mesure GeHP). Dans le mélange ${ }^{239} \mathrm{Pu} /{ }^{241} \mathrm{Am}$, l'activité de ${ }^{241} \mathrm{Am}$ représente $10 \%$ de celle de ${ }^{239} \mathrm{Pu}$ (Razafindralambo, 1995).

Interference of ${ }^{239} \mathrm{Pu} U X L$ and ${ }^{241} \mathrm{Am} \mathrm{NpXL}$ rays (HPGe measurement). In the mixture ${ }^{239} \mathrm{Pu} /{ }^{24 I} \mathrm{Am}$, the activity of ${ }^{24 I} \mathrm{Am}$ represents $10 \%$ of this of ${ }^{239} \mathrm{Pu}$ (Razafindralambo, 1995). 


\section{Nouvelles orientations de recherches}

Se fondant sur les données de référence fournies par les détecteurs $\mathrm{Ge}(\mathrm{HP})$, les critères auxquels doit répondre un nouveau système de spectrométrie in vivo pour obtenir un gain de performance significatif ont été établis en première analyse comme suit :

- une résolution en énergie équivalente à celle d'un détecteur $\mathrm{Ge}(\mathrm{HP})$ pour la spectrométrie $X$,

- un matériau et une structure de détecteur donnant une faible réponse au bruit de fond radiatif,

- une surface de détection importante permettant d'ajuster la géométrie de détection au volume pulmonaire,

- une efficacité suffisante pour pouvoir détecter la raie $\gamma$ de ${ }^{241} \mathrm{Am}$.

Les différentes voies de recherche qui ont été explorées par différents auteurs en suivant ces différents critères comportaient principalement : (i) la production de cristaux de germanium de grand volume permettant le développement de systèmes in vivo de plus grande surface. Cependant, 1'utilisation de cryostats restera toujours un réel problème (Twomey et Keyser, 1992); (ii) l'utilisation de nouveaux mélanges de gaz permettant une meilleure efficacité de détection. Actuellement, il n'est cependant pas encore démontré qu'une bonne résolution pourra être alliée à une bonne efficacité nécessaire pour la mesure des actinides (Toohey et al., 1991; Razafindralambo, 1995); (iii) et enfin la recherche de nouveaux semi-conducteurs fonctionnant à température ambiante. Cette dernière voie semble être la plus prometteuse pour le futur de la mesure in vivo (Toohey et al., 1991). La raison principale est que le recours à une technologie à température ambiante présente l'avantage vis-à-vis du détecteur germanium fonctionnant à température azote liquide de permettre une grande surface de détection mieux ajustée au thorax (Selby et al., 1995b; Razafindralambo, 1995).

Parmi les différents matériaux deux types de semi-conducteurs se sont avérés comme très prometteurs pour l'anthroporadiamétrie car répondant bien aux critères précités: le tellure de cadmium (CdTe) et le silicium.

L'application du CdTe pour la spectrométrie X et $\gamma$ est étudiée depuis quelques années dans un certain nombre de laboratoires (Verger et al., 1996). Il présente, en effet, l'avantage par rapport au Si et même au Ge d'être plus dense et donc d'offrir des efficacités de détection intrinsèques supérieures. Cependant, en raison de problèmes de fabrication, une résolution acceptable ne peut être obtenue actuellement que pour de très petits volumes, limitant leur utilisation actuelle en spectrométrie au niveau du laboratoire et à des systèmes de faibles dimensions (Scheiber, 1996). Des recherches importantes sont actuellement réalisées dans ce domaine et pourraient dans le futur conduire à la pro- 
duction de cristaux de grande taille et ainsi ouvrir leur application en anthroporadiamétrie.

Le silicium de haute résistivité a été identifié comme le plus intéressant sur le plan de la maturité technologique (de Carlan et al., 1996). Comme le germanium, il appartient à la catégorie des détecteurs qui permettent potentiellement d'obtenir une très bonne résolution en énergie contrairement aux détecteurs gazeux et aux scintillateurs. De plus, les progrès de la technologie planaire sur silicium passivé implanté de faible capacité ont conduit à la réalisation de détecteurs à dérive ayant des bruits électroniques de plus en plus faibles (Strüder, 1989) et donc des résolutions excellentes, équivalentes à celle des GeHP, voire meilleures.

Deux difficultés majeures impliquent cependant de démontrer la faisabilité du silicium pour une application telle que l'anthroporadiamétrie pulmonaire. La difficulté principale reste l'utilisation de détecteurs fiables sur le plan de la technique de fabrication et conservant leur niveau de qualité sur une grande surface. Elle nécessite, en particulier, une électronique associée intégrée très bas bruit permettant une gestion d'un grand nombre de voies. On peut néanmoins espérer, grâce aux progrès réalisés en matière de micro-électronique et grâce à la collaboration avec des laboratoires spécialisés, de surmonter ces problèmes dans les prochaines années. La seconde difficulté est la faible gamme d'énergie accessible au silicium. Les premières simulations des performances de ce type de détecteurs ont montré qu'un facteur 2,8 pouvait être gagné sur la limite de détection pour la mesure de ${ }^{239} \mathrm{Pu}$ par rapport aux systèmes germanium utilisés actuellement (Razafindralambo, 1995). Cette étude montre, cependant, que la mesure peut être raisonnablement étendue aux rayonnements gamma de $60 \mathrm{keV}$ de ${ }^{241} \mathrm{Am}$ avec une augmentation acceptable de la LD (facteur 2). En tenant compte de l'application spécifique à la mesure pulmonaire, un premier système de détection mettant en jeu des modules de 3 détecteurs Si superposés et une électronique associée spécialement adaptée a été développé (de Carlan et al., 1996). Les mesures effectuées à ce jour sur sources étalées ont confirmé ces simulations (de Carlan et al., 1997). En particulier, les premiers résultats sur la réduction du bruit de fond par anticoïncidence interne ont permis d'abaisser le bruit de fond d'un facteur 3 conduisant à des gains en limite de détection par rapport au détecteurs GeHP d'un facteur 1,75 au niveau des raies $\mathrm{X}$ de basse énergie. Sur ces bases, l'étape suivante consistera à construire un système anthroporadiamétrique réaliste de grande surface et d'établir les performances que l'on peut obtenir pour la détection pulmonaire d'actinides tels que ${ }^{239} \mathrm{Pu}$ et ${ }^{241} \mathrm{Am}$.

Remerciements. Nous remercions tout particulièrement MM. Mazur, Cahan et Rouger du DAPNIA (CEA/DSM, Saclay) et M. Burger de la société CANBERRA (Olen, Belgique) pour leur contribution dans le projet d'étude et le développement du nouveau système de détecteurs au silicium (ANTHRO-Si). 


\section{MESURES ANTHROPORADIAMÉTRIQUES DANS LES BASSES ÉNERGIES}

\section{RÉFÉRENCES}

Bataller G. et Girard de Vasson O. (1993) Mesure de la charge pulmonaire en américium et plutonium avec un ensemble de huit détecteurs germanium basse énergie. In Proc. Journées de spectrométrie gamma et X 93 (St Rémy les Chevreuses, France). DTA/DAMRI ed., 1994, Rapport CEA-N-2756, 253-258.

Bérard Ph. et Aussel J.P. (1993) Surveillance anthroporadiamétrique X et gamma des travailleurs exposés aux composés de l'uranium à l'aide de détecteurs coaxiaux. In Proc. Journées de spectrométrie gamma et X 93 (St Rémy les Chevreuses, France). DTA/DAMRI ed., 1994, Rapport CEA-N-2756, 259-263.

Bland C.J., Morel J., Etcheverry E. et Lépy M.C. (1992) Determination of ${ }^{239} \mathrm{Pu}$ et ${ }^{241} \mathrm{Am}$ LX-ray intensities using a Simplex method for fitting peaks. Nucl. Instr. and Meth., A312, 323-333.

Currie L.A. Limits for Qualitative Detection and Quantitative Determination. (1968) Anal. Chem., 40, 486-488.

Daburon M.L. et Rigaudière R. (1976) Détecteur Phoswich (INa-ICs) pour la détection X du ${ }^{239} \mathrm{Pu}$ et de l'24i Am dans les poumons. Rapport CEA-R-4794.

de Carlan L., Burger P., Cahan B., Franck D., Mazur C., Passerieux J.P. et Pihet P. (1996) Discrete analog electronics for high resistivity silicon detectors used for $\mathrm{X}$ and $\gamma$ ray spectrometry in whole body monitoring. Nucl. Inst, and Meth., A380, 371-375.

de Carlan L., Franck D., Beaujard P., Burger P., Cahan B., Maillard O., Mazur C., Pihet P, et Soulié R. (1997) Investigation of passivated silicon detectors for direct measurements of actinides. Radioanalytical \& Nuclear Chemistry, 22, $\left.n^{\circ}\right]-2$.

Ehret R., Kiefer H., Maushart R., Mohrle G. (1964) Performance of an arrangement of several large area proportional counters for the assessment of Pu239 lung burdens. Symposium « Assessment of radioactivity in man», Heidelberg, 11-16 May 1964, IAEA, Vienne, Vol 1, 141-149.

Falk R.B., Tyree W.H., Wood C.B. et Lagerquist C.R. (1979) A system of high purity germanium detectors for the detection and measurement of inhaled radionuclides, In : IAEA Symp. Advances in Radiation Protection Monitoring, IAEA, Vienne, 445-455.

International Commission on Radiological Protection (ICRP). (1994) Human respiratory tract model for radiological protection. ICRP Publication 66. Annals of the ICRP, $24 \mathrm{n}^{\circ} 1-4$.

International Commission on Radiological Protection (ICRP). (1995) Dose coefficients for intakes of radionuclides by workers. ICRP Publication 68. Replacement of ICRP Publication 61.

Kang C., Newton D., Warner A.J., Absolom T.A., Kruchten d.A., Anderson A.L. et palmer H.E. (1993) A comparisonof techniques in the assessment of chest wall thickness and composition. Health Physics, $64 \mathrm{n}^{\circ} 4,406-411$.

Morucci J.P. (1966) Nouveau compteur proportionnel destiné à la détection in vivo des traces de plutonium dans les poumons. Rapport CEA-R-3027.

Newton D., Fry F.A., Taylor B.T., Eagle M.C. et Sharma R.C. (1978) Interlaboratory comparison of techniques for measuring lung burdens of low-energy photon-emitters. Health Physics, 35,751 771 .

Palmer H.E., Rieksts G.A. (1984) The use of planar high-purity Ge detectors for in vivo measurement of low-energy photon emitters. Health Physics, $47 \mathrm{n}^{\circ} 4,569-578$.

Pihet P., Beau P., Bérard Ph., Franck D., Malarbet J.L., Razafindralambo N. et Rannou A. (1995a) Low energy spectroscopy for monitoring lung retention in respect « Radiation Dose Management » Windermere, UK (9-11 Octobre 1995). 
Pihet P., Burger P., Franck D., Bérard P., Le Noir de Carlan L., Paul D., et Razafindralambo N. (1995b) Perspectives of silicon detectors for $X$ and low energy gamma ray spectroscopy in whole body counting. Rad. Prot. Dosim., 61, 149-152.

Ramsden D. et Foster P.P. (1986) Assessment of plutonium in the lung. Atom, 361.

Razafindralambo N. (1995) Limite de détection de la spectrométrie X pour l'anthroporadiamétrie pulmonaire du plutonium: Analyse et perspectives de développement. Thèse de l'université Paul Sabatier.

Razafindralambo N., Franck D., Morel J. et Pihet P. (1994) Applicabilité de l'analyse spectrale globale pour l'anthroporadiamétrie pulmonaire de ${ }^{239} \mathrm{Pu}$. In : Proc. Journées de spectrométrie gamma et X 93 (St Rémy les Chevreuses, France, 1993). DTA/DAMRI ed., Rapport CEA-N-2756, 283288 .

Rundo J., Strauss M.G., Sherman I.S. et Brenner R. (1978) Methods for the assay of plutonium in vivo: What are the alternatives? Health Physics, 35, 851-858.

Scheiber C. (1996) New developments in clinical applications of CdTe And CdZnTe. Nucl. Inst. and Meth., A380, 385-391

Selby J., Lardy M. M., Carbaugh E. H., Lynch T. P. et Strom D. J. (1994) Internal dosimetry monitoring equipment: present and future. Radiat. Prot. Dosim., 53, n 1-4, 49-58.

Strüder L. (1989) Recent development in semiconductor detectors and on-chip electronics. Nucl. Inst. Meth., A283, 387-398.

Toohey R., Palmer E., Anderson L., Berger C., Cohen N., Eisele G., Wachhoz B. et Burr W. (1991) (Whole-body Counting Working Group). Current status of whole-body counting as a means to detect and quantify previous exposures to radioactive materials. Health Physics, 60, Sup 1, 7 42 .

Twomey T.R. et Keyser R.M. (1992) Advances in large-diameter, low-energy HPGe detectors. Nucl. Instr. and Meth., A312, 78-86.

Verger L., Baffert N., Rosaz M. et Rustique J. (1996) Characterization of CdZnTe and CdTe : Ci materials and their relationship to $\mathrm{X}$ - and g-ray detector performance. Nucl. Inst. and Meth., $\mathbf{A 3 8 0}$, 121-126. 